\title{
因HAD
}

ISSN-L: 2530-5115

DOI: http://doi.org/10.22585/hospdomic.v1i1.4

\section{Nutrición enteral domiciliaria prescrita en el Hospital Universitario Infanta Cristina de Badajoz (España)}

\section{Home enteral nutrition prescribed in Infanta Cristina University Hospital in Badajoz (Spain)}

Luis Miguel Luengo Pérez', Sara Guerra Martínez², Laura Monica Georgescu³

1. Departamento de Ciencias Biomédicas, Facultad de Medicina, Universidad de Extremadura. Badajoz, España.

2. Departamento de Ciencias Biomédicas, Facultad de Medicina, Universidad de Extremadura. Badajoz, España.

3. Facultad de Medicina y Farmacia de la Universidad de Oradea, Rumanía.

Correspondencia/Correspondence

luismiluengo@yahoo.es

Recibido/Received

22.11.2016

Aceptado/Accepted

29.11 .2016
Conflicto de Intereses/Competing interest No existe ningún conflicto de interés en el presente estudio.

Agradecimientos/Acknowledgments

Queremos agradecer al personal de enfermería de la Unidad de Nutrición la ayuda prestada en la asistencia a los pacientes con nutrición enteral domiciliaria. pital Universitario Infanta Cristina de Badajoz (España). Hosp Domic. 2017;1 11):35-45. 


\section{RESUMEN}

Objetivo: Conocer la práctica real de la Nutrición Enteral Domiciliaria en la Unidad de Nutrición del Hospital Universitario Infanta Cristina de Badajoz durante 12 meses y, de forma secundaria, comparar los datos existentes con otros centros a nivel nacional e internacional.

Método: Estudio descriptivo, observacional y retrospectivo de los pacientes atendidos por la Unidad de Nutrición, en el ámbito de la nutrición enteral domiciliaria, durante 12 meses consecutivos.

Resultados: El número de pacientes mayores de 14 años con NED durante los 12 meses del estudio fue de 176 (52\% varones). La edad media fue de 71,3 años (17-104) y la duración media del soporte de 10 meses $(307,16$ días), con causa de finalización más frecuente el fallecimiento $(78,2 \%)$. La enfermedad de base que se registró con más frecuencia fue la neurológica en 97 pacientes $(55,1 \%)$, seguida de la neoplásica en 55 (31,3\%). La vía de administración más utilizada fue la sonda nasogástrica $(69,3 \%)$, seguida de la gastrostomía (14,2\%) y la oral (10,8\%). El $42,05 \%$ de los pacientes presentaban una actividad limitada y el 30,1\% estaba confinado en cama/sillón. El 73,3\% de los adultos requerían ayuda total o parcial. El suministro de la fórmula se hacía desde las farmacias de referencia, mientras que el material fungible se suministraba desde Atención Primaria en el 78,4\% y desde el hospital en el resto.

Conclusión: El número de pacientes registrados aumenta en relación con los años anteriores, pero se mantienen diferencias respecto a registros a nivel nacional e internacional.

Palabras clave: Nutrición Enteral; Métodos de Alimentación; Atención Ambulatoria; Registros como Asunto; Sistema de Registros.

\section{ABSTRACT}

Aim: To know the real practice of Home Enteral Nutrition at the Nutrition Unit of Infanta Cristina University Hospital in Badajoz for 12 months and, secondarily, to compare it with data published from other national and international centres.

Methods: Retrospective, observational and descriptive study of the patients visited by the Nutrition Unit concerning Home Enteral Nutrition for 12 consecutive months.

Results: The number of patientes older than 14 with Home Enteral Nutrition during the 12 months of the study was 176 (52\% males). Mean age was 71.3 years (17-104) and mean duration of nutritional support was 10 months (307.16 days), with death as the most common cause of finishing it (78.2\%). Neurological disease was the most commonly registered cause, in 97 patients $(55.1 \%)$, followed by neoplastic disease in 55 (31.3\%). The most common access route was nasogastric tube (69.3\%), followed by gastrostomy (14.2\%) and per os (10.8\%). 42.05\% of the patients had limited activity and $30.1 \%$ were bed-to-chair; $73.3 \%$ required complete or partial help. Nutritional formula was suplied from Pharmacy offices, while material was given in Primary Care to $74.8 \%$ of the patients and in Hospital to the rest.

Conclusion: The number of patients registered has been increased comparing with the previous years, but differences from other national and international registries remain.

Keywords: Enteral Nutrition; Feeding Methods; Ambulatory Care; Records as Topic; Registries. 


\section{INTRODUCCIÓN}

El continuo avance de la medicina hace que cada vez exista mayor número de pacientes crónicos, con muy diversas patologías de base, incapaces de hacer frente a sus necesidades de energía y nutrientes mediante el consumo de alimentos naturales. El aporte adecuado de nutrientes es de suma importancia, ya que tanto la sobrenutrición como la desnutrición pueden agravar el estado clínico, siendo el organismo incapaz de mantener las funciones vitales. A su vez, se ven afectados los mecanismos de defensa, cicatrización y reposición de tejidos, y como consecuencia se produce una menor tolerancia y respuesta frente a los distintos tratamientos (1-3).

Cuando se dan estas circunstancias es necesaria la indicación de distintos tipos de soporte nutricional artificial con la finalidad de cubrir las necesidades del organismo, aportando los nutrientes precisos de forma cuantitativa y cualitativa (2). La nutrición artificial consiste en la administración de los nutrientes y demás agentes terapéuticos adjuntos, bien a través de la vía digestiva (nutrición enteral: NE) o bien mediante la vía intravenosa (nutrición parenteral: NP) (1).

Será candidato de NE aquel paciente que, disponiendo de una función gastrointestinal suficiente para asimilar los nutrientes, esté desnutrido o presente riesgo de estarlo porque la ingesta oral de alimentos (incluso suplementada) es inadecuada o insuficiente. Así mismo, será candidato a NP todo paciente que no posea un sistema gastrointestinal funcionante para recibir los nutrientes necesarios, a fin de cubrir los requerimientos nutricionales (1).

La nutrición artificial domiciliaria (NAD) es una modalidad terapéutica que está en aumento por todas las ventajas que implica, ya que generalmente son tratamientos que deben ser administrados por largos períodos de tiempo. Esto posibilita que el paciente esté integrado en su entorno sociofamiliar permitiendo el desarrollo de sus actividades cotidianas. Además, disminuye la probabilidad de presentar complicaciones relacionadas con la estancia hospitalaria (infecciones nosocomiales), consiguiendo un mayor grado de satisfacción y mejora de la calidad de vida, al mismo tiempo que permite una reducción de los costes sanitarios (4).

El enorme crecimiento que ha tenido la NAD en las últimas décadas ha hecho necesario el desarrollo de registros de pacientes que se benefician de este tratamiento. Gracias a estos registros podemos aproximarnos a conocer la prevalencia de la nutrición enteral (NED) y parenteral domiciliaria (NPD) en distintos países, las indicaciones más frecuentes, las complicaciones principales, la supervivencia y calidad de vida, así como datos sobre los modos de financiación. Esta información puede aplicarse en diferentes ámbitos, persiguiendo una mejora de la calidad asistencial (5).

La importancia de los registros está determinada por la calidad de los datos que los componen, pero conseguir información que muestre un fiel reflejo de la realidad asistencial de la NAD no es tarea fácil, ya que, en general, los registros no son obligatorios y dependen de la voluntad individual de cada colaborador $(6,7)$.

En España, la información de la que se dispone es la facilitada por el Grupo de Trabajo de Nutrición Artificial Domiciliaria y Ambulatoria (NADYA), de la Sociedad Española de Nutrición Parenteral y Enteral (SENPE), que se creó en 1992 y, desde su inicio, ha estado continuamente esforzándose para que los profesionales implicados en este tratamiento se sientan animados y estimulados a registrar sus pacientes y episodios con el máximo rigor (7). El registro NADYA, dado que es un registro voluntario y no incluye todos los centros ni prescriptores, infraestima el consumo de nutrición artificial domiciliaria en España.

\section{Los objetivos de este trabajo son:}

Objetivo principal: Analizar las características de las prescripciones realizadas en la Unidad de Nutrición Clínica y Dietética del Hospital Universitario Infanta Cristina de Badajoz, en el año 2013. 
Objetivo secundario: Comparar los datos anteriores con los existentes de otros centros a nivel nacional e internacional.

\section{MÉTODOS}

\section{Recogida de datos}

Se recogieron los datos de los pacientes a los que se prescribió nutrición artificial ambulatoria en la Unidad de Nutrición Clínica y Dietética del Hospital Universitario Infanta Cristina de Badajoz (Extremadura) durante 12 meses consecutivos. Los pacientes que se contabilizaron fueron todos aquellos no hospitalizados que recibían tratamiento con al menos $1000 \mathrm{kcal} /$ día mediante fórmulas de nutrición enteral por cualquier vía (oral, sonda nasoentérica u ostomías).

Además de los datos sociodemográficos del paciente, se recabaron los referentes a la patología de base, la vía de acceso de la nutrición artificial, el nivel de actividad, el grado de autonomía, la forma de suministro del producto y del fungible así como las características del episodio de tratamiento, como es la fecha de inicio y de finalización, incluyéndose el motivo de la misma.

Estos datos se incluyeron en la base de datos del registro NADYA de la SENPE. Se trata de un registro en red al que se accede mediante una clave y contraseña a través de la página web del mismo grupo (www.nadya-senpe.com).

\section{Análisis descriptivo}

A partir del registro NADYA, que permite la realización de la exploración estadística de los datos introducidos, se realizó el estudio descriptivo de los datos. No obstante, para el cálculo de las frecuencias absolutas y relativas de las variables así como algunas medidas descriptivas o estadísticos se empleó el paquete estadístico SPSS versión 21.0, que además, permitió la obtención de los datos más relevantes en forma de tablas y figuras.

En el análisis de los datos únicamente se consideró la población adulta superior a los 14 años de edad.

A partir de este análisis, se compararon los resultados obtenidos con los registros existentes de otros centros a nivel nacional e internacional, sabiendo que cada investigador o usuario registrado en las bases de datos (en España, NADYA), sólo tiene acceso a los datos correspondientes a su propio centro, garantizándose la confidencialidad de los mismos de acuerdo a la normativa vigente, la Ley Orgánica 15/1999, de 13 de diciembre, de Protección de Datos de Carácter Personal (7).

\section{RESULTADOS}

Durante los 12 meses evaluados, se registraron 176 pacientes con nutrición enteral domiciliaria y ambulatoria, en la Unidad de Nutrición Clínica y Dietética del Hospital Universitario Infanta Cristina de Badajoz. Esto supone una prevalencia de 692,9 pacientes con NED por millón de habitantes para ese año, teniendo en cuenta el número total de pacientes registrados en NADYA y los datos oficiales de población del Área de Salud de Badajoz.

Se administró NED a un total de 91 varones (51,7\% de los pacientes) y de 85 mujeres (48,3\%). La edad media fue de 71,3 años (17-104), siendo todos los pacientes de edad superior a 14 años, 
y encontrándose el mayor número de los pacientes del registro $(47,7 \%)$ en el grupo de edad de más de 74 años.

Las enfermedades que motivaron el uso de la NED fueron muy numerosas, en donde la patología de base más frecuentemente registrada fue la neurológica en 97 pacientes $(55,1 \%)$, seguida en $67(38,1 \%)$ de enfermedades neoplásicas. La cirugía ORL y maxilofacial así como la estenosis esofágica no tumoral fueron la causa de $2(1,1 \%)$ de las indicaciones en ambos casos, y el trastorno severo de la motilidad intestinal junto con otras causas completan el conjunto de las indicaciones (Figura 1).

Figura 1. Patologías que justifican la indicación de nutrición enteral domiciliaria, por sexos.
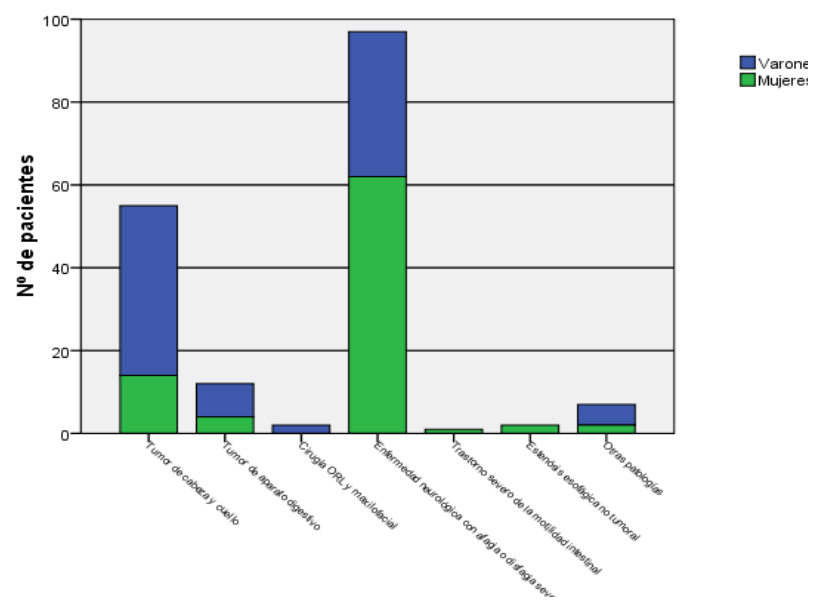

La vía de administración más utilizada en los pacientes del registro fue la sonda nasogástrica en $122(69,3 \%)$ pacientes, seguida de gastrostomía en $25(14,2 \%)$, la oral $19(10,8 \%)$ y en $10(5,7 \%)$ casos la yeyunostomía (Figura 2).

Figura 2. Vías de acceso empleadas.

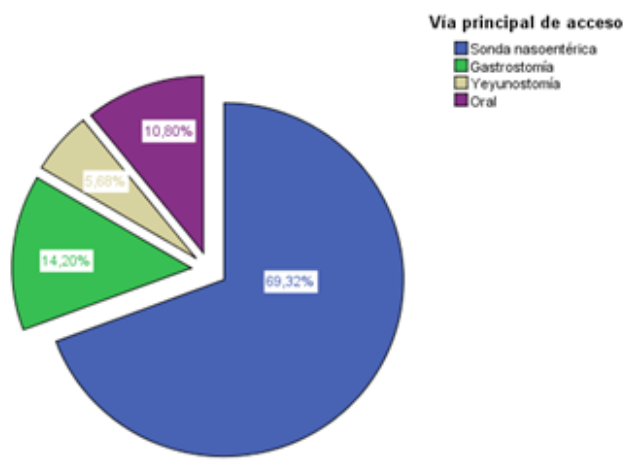


La duración media del soporte con nutrición enteral en los pacientes fue de 10 meses $(307,16$ días), siendo la duración entre 12 y 24 meses en un 31,3\% de los usuarios. Durante el período de estudio, finalizaron 101 episodios (57,4\% del total), siendo los motivos más frecuentes de la finalización del tratamiento el fallecimiento del paciente en el $78,2 \%$ de los casos y el paso a vía oral en el $18,8 \%$ (Figura 3 ).

Figura 3. Duración de los episodios.

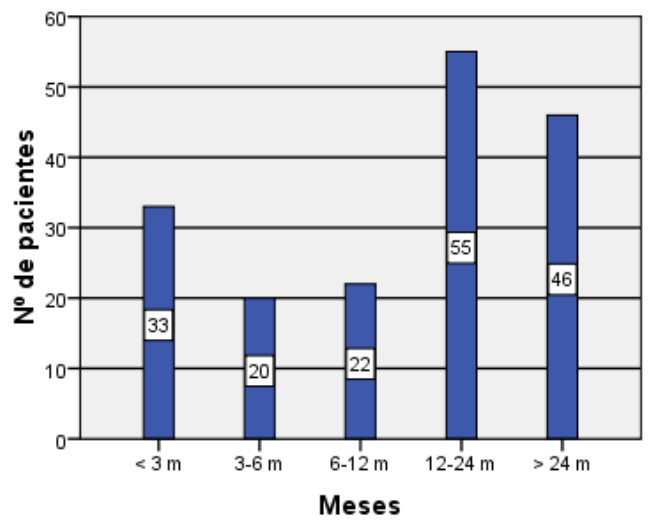

El nivel de actividad de la mayoría de los pacientes estaba limitado (74 pacientes, 42,05\%). La actividad en los demás casos era normal en 49 pacientes $(27,8 \%)$ y el resto, 53 , estaban limitados a cama-sillón (30,1\%). Del total de pacientes, sólo el $26,7 \%$ eran independientes ( 47 casos) y totalmente dependientes 67 (38,1\%), requiriendo ayuda parcial $62(35,2 \%)$.

El suministro de la fórmula de nutrición enteral se realizó por la farmacia de referencia en la totalidad de los pacientes. En cuanto a la provisión de material fungible en aquellos pacientes que precisaban el uso de sondas, se efectuó a través de atención primaria en 138 usuarios (78,4\%), el hospital en 29 de los casos (16,5\%) y solamente $8(4,6 \%)$ no precisaban.

\section{DISCUSIÓN}

La nutrición enteral domiciliaria ha sufrido un gran crecimiento en los últimos años, aunque su evolución ha sido muy diversa ya que el ritmo de crecimiento no es igual en todos los países. La obtención de datos reales sobre la incidencia es muy complicado debido a la ausencia de registros representativos, en parte ocasionado por la ausencia de obligatoriedad en la creación de los registros, así como la variabilidad de las políticas sanitarias $(1,8)$.

\section{Datos de centros españoles}

La práctica de la NED en España está legislada por el Real Decreto 1030/2006, de 15 de septiembre, por el que se establece la cartera de servicios comunes del Sistema Nacional de Salud y el procedimiento para su actualización. A pesar de la existencia de legislación, su organización 
y funcionamiento dependen no sólo de cada comunidad autónoma, sino incluso de cada centro hospitalario.

Esta situación da lugar, por ejemplo, a que algunas comunidades autónomas suministran las fórmulas de nutrición y el material necesario en las farmacias de los centros hospitalarios mientras que en otras son las oficinas de farmacia las responsables.

Con respecto a la información de la que se dispone en España referente a los datos de NAD, como ya se ha mencionado a lo largo del trabajo, es la facilitada por el Grupo de Trabajo de Nutrición Artificial Domiciliaria y Ambulatoria (NADYA), de la Sociedad Española de Nutrición Parenteral y Enteral (SENPE). No obstante, al tratarse de un registro voluntario, los datos que genera no son un reflejo fiel de la realidad.

1. Prevalencia: el incremento de la prevalencia de empleo de la NED es constante año tras año, ya que desde 1992 el número de pacientes registrados se ha multiplicado por 10 y el de centros participantes en el registro nacional por dos (9). En los datos parciales de distintos territorios del país podemos observar la mayor prevalencia en todos los registros hasta el momento, siendo la incidencia en el Área de Salud de Badajoz de 216,5 por millón de habitantes en el año 2007 y de 692,9 en 2013, cifras superiores en La Coruña con 870,2 casos por millón de habitantes (10) e inferiores para Barcelona con 436,1 casos (11), León y Valladolid, con 258,1 y 296, respectivamente $(12,13)$.

2. Patología de base: la principal enfermedad que motivó la instauración de la NED en Badajoz para el año 2013 fue la neurológica, seguida de la enfermedad neoplásica (55,1\% y 38,1\%, respectivamente) al igual que sucedió para el año 2007 (56,4\% y 27,2\%), y manteniéndose el mismo patrón de distribución en Galicia en 1999 (43,6\% y 30,7\%) y 2006 (39,7\% y 32,1\%) (10,14), León en 2008 (51\% y 16\%) (12), Barcelona en 2007 (90,3\% y 1,7\%) (15), aunque opuesto para Barcelona en 1999 en dónde la principal patología fue la neoplasia (44\%) y las enfermedades neurológicas (28\%) (11), al igual que en Madrid (41\% y 38\%) (16). Las diferencias encontradas pueden deberse fundamentalmente al tipo de entorno en que se analiza la actividad (pacientes institucionalizados o no), la actividad de los distintos hospitales o la clasificación del tipo de pacientes (3).

3. Vía de administración: la vía de acceso más frecuentemente empleada que se refleja en la mayor parte de los estudios españoles como son los de Barcelona, Galicia, León y Valladolid $(10,14)$ es la vía oral, no resultando similar a los resultados obtenidos en nuestro estudio para 2013, en dónde la sonda nasogástrica fue el método más empleado para ese año. El Sistema Sanitario Público español financia la prescripción de la nutrición enteral-oral (a diferencia de otros entornos geográficos) lo que influye directamente como causa fundamental de las altas cifras de soporte a través de la vía oral (3). No obstante, es un dato difícil de contrastar ya que todavía existen estudios en los que sólo se contabilizan a los pacientes que reciben la nutrición enteral a través de sondas (15), en parte relacionado con la tendencia de no incluir a los pacientes con NE por la vía oral debido a la sobrecarga de trabajo que supone y a los sesgos aportados por los pacientes con suplementación nutricional, priorizando a los pacientes que reciben la NE a través de sondas (9).

4. Evolución del tratamiento: la duración media del tratamiento en los pacientes ha ido aumentando en los últimos años y aunque los motivos principales de finalización siguen siendo los mismos, el fallecimiento en la mayoría de los casos y el paso a vía oral en segundo lugar $(11,14)$, se observa una tendencia al aumento de la frecuencia de esta última. Sin embargo, a pesar de que los datos no difieren de manera importante con respecto a los de otros estudios nacionales, llama la atención los datos de La Coruña en el año 2006, donde se describe que la principal causa de suspensión fue la falta de seguimiento $(45,3 \%)$, seguida de la muerte del paciente (30\%) (10). La defunción como motivo principal de la finalización puede parecer un resultado 
negativo del proceso, no obstante hay que destacar que la muerte (en la mayor parte de los casos) se asocia con la patología de base y la edad del paciente, y no con una incorrecta indicación del tratamiento, ya que se alcanzan a corto plazo los principales objetivos de la NED: mejorar la calidad de vida y evitar las hospitalizaciones (8).

5. Suministro de producto y fungible: el modo de obtención de fórmulas y demás tipo de material refleja las distintas formas de distribución en nuestro país, ligado al desarrollo de la normativa de NED en las distintas Comunidades Autónomas. En Cataluña y Galicia, se distribuye desde los hospitales, ya sea a domicilio (Cataluña) o desde las farmacias hospitalarias (Galicia), mientras que en el resto las fórmulas se dispensan en las oficinas de farmacia $(3,8)$.

\section{Datos en centros internacionales}

La práctica de la NED es muy diferente entre los distintos países, mientras algunos como Estados Unidos o Italia están alcanzando cotas altas, en otros es una práctica todavía poco difundida. Así mismo, la legislación y organización difiere de unos países a otros, ya que en algunos países como Estados Unidos y Reino Unido, existen unas normativas estándar aplicables a nivel nacional, pero no ocurre lo mismo con otros en los que ni siquiera hay legislación específica para este tratamiento, tal es el caso de Austria, Croacia, Alemania o Polonia (1).

Al igual que sucede en España, existen registros de NAD que comparten algunas características con el registro NADYA de nuestro país (17) como el registro OASIS (Oley Foundation Register) de la sociedad científica (Oley-ASPEN Society of Parenteral and Enteral Nutrition) en Estados Unidos y Canadá, que se creó como un registro voluntario de pacientes con NAD y se suspendió en 1993, por lo que sólo existen datos correspondientes a los años 1985-1992. El registro BANS (British Artificial Nutrition Survey) en Reino Unido nos ofrece anualmente los datos de pacientes (adultos y niños) con nutrición enteral y parenteral. El registro SINPE de la Sociedad Italiana de Nutrición Parenteral y Enteral en Italia. El Japanese HPN Registry de la Sociedad Japonesa de Nutrición Parenteral y Enteral en Japón. El registro BDR WA (Birth Defects Register of Wester Australia) ofrecido en Australia por la PMHC durante diez años 1986-1996. El GEMIDES Datenbank de la FACGF en Alemania.

En general, podemos afirmar que la práctica clínica de la NAD está muy extendida, pero con gran variabilidad en donde no encontramos guías de actuación común y son escasos los estudios multicéntricos. A continuación, se expone una descripción comparativa del uso de esta práctica a nivel mundial a partir de los datos obtenidos.

1. Prevalencia. Al igual que sucede en España, es difícil conocer la incidencia exacta en otros países. En parte, esto es debido al propio concepto o consideración de la NED, puesto que no existe un acuerdo en la cantidad mínima del tratamiento o la vía de acceso utilizada. Así, mientras algunos registros sólo tienen en cuenta la nutrición a través de sonda nasoentérica u ostomía (Francia, Italia y Reino Unido), en otros también se considera la vía oral al superar una cantidad determinada de calorías de la dieta (Bélgica, Dinamarca, España, Israel, Polonia y República Checa) $(1,3,18)$. Si consideramos algunos datos publicados en diversos estudios, encontramos un aumento de la prevalencia en general, tal es el caso de la región de Campania en Italia, en dónde el aumento es del 321,1\%, pasando de los 199 pacientes en el año 2005 a los 838 en 2012, siendo la incidencia en 16 regiones de Italia de un total de 20 para el año 2005 de 152,6 casos por millón de habitantes (6955 casos) $(19,20)$. En el informe anual de BANS de 2011 se estiman 798 casos por millón de habitantes, cifra mayor del informe de 2007 en dónde se contabilizaban unos 500 casos (21). Otro estudio sobre la incidencia de NED en Francia nos aporta una prevalencia de 573 casos por millón de habitantes (22) para el año 2012, cifras 
superiores a las aportadas por el grupo NADYA para ese mismo año, donde se registraron 2695 pacientes de 29 hospitales, lo que supone una prevalencia de 57,58 pacientes/millón de habitantes (23).

2. Patología de base: las principales diferencias existentes entre distintos países se deben más al sistema de registro (sólo NED por sonda frente a cualquier vía de administración) que a diferencias en la indicación. Las enfermedades neurológicas seguidas de las neoplásicas son las más frecuentes (19). En el estudio italiano de la SINPE, la enfermedad neurológica afecta al $79,9 \%$ de los pacientes y en segundo lugar los oncológicos, datos coincidentes con el estudio de la región de Campania en donde la enfermedad neurológica afecta a un $80 \%$ del total $(19,20)$. En el informe de BANS la enfermedad neurológica ocupa el primer puesto seguida por las neoplasias, siguiendo el mismo patrón que en el resto de países; no obstante, se aprecia un descenso de la incidencia del 58,4\% de casos en el año 2000 a 45,7\% en 2010. Esto es debido al aumento de los casos de cáncer en un 14,2\% del total en los diez años (21).

3. Vía de administración: la vía de administración a través de gastrostomía es el método más frecuentemente registrado en la mayoría de los países, debido fundamentalmente a la falta de registros en dónde se incluya los pacientes que reciben nutrición enteral-oral y por la falta de financiación de esta vía que da lugar a un mayor empleo de ostomías de las que pueden estar justificadas $(8,19)$. En el registro español para el año 2011, la principal vía utilizada es la sonda nasogástrica y en segundo lugar la gastrostomía, coincidiendo con los resultados obtenidos en Badajoz, pero no así con otras comunidades autónomas en dónde la vía oral ocupa el primer puesto (25). No obstante, no se pueden considerar estos datos representativos ya que el grupo NADYA detectó para el año 2010 una falta de cumplimentación de la vía de acceso en un 90\% de los casos dando lugar a un falseo de los resultados (9).

4. Evolución del tratamiento: los resultados no difieren en los distintos países, coincidiendo el fallecimiento relacionado con la enfermedad de base y el paso a vía oral los motivos más frecuentes de la finalización del tratamiento. Así se muestra en el estudio de BANS, en donde el $41,4 \%$ de los casos finalizan por fallecimiento y el 20,7\% pasan a vía oral. Además, el descenso en el número de pacientes neurológicos en los últimos años ha supuesto un aumento de pacientes con actividad normal del 17\% al 40\% en 2010 (siendo el ascenso del 10\% en los últimos dos años), y por tanto, un descenso de pacientes dependientes (21).

5. Suministro de producto y fungible: en algunos países, como Francia y Dinamarca, todo el material accesorio que necesita el paciente para su tratamiento es suministrado por los hospitales; en otros, como Alemania o Austria, son las industrias las que se ocupan de su distribución, y finalmente, en algunos países, como Italia y el Reino Unido, existen las dos modalidades (1).

Podemos concluir que el uso de la NED es muy variable tanto a nivel nacional como, sobre todo, internacional. La prevalencia de empleo de NED es la mayor que existe hasta el momento, multiplicándose por 10 el número de pacientes desde el año 1992 en España. La prevalencia de NED en España es alrededor de cinco veces inferior a la de otros países. Las enfermedades más frecuentes que motivan el uso de la NED son la neurológica, seguida de las enfermedades neoplásicas. La vía de NE más empleada en nuestro medio es la sonda nasogástrica, seguida de la gastrostomía. El motivo de finalización más frecuente de la NED es el fallecimiento, seguido del paso a vía oral, al igual que en los estudios publicados. 


\section{BIBLIOGRAFÍA}

1. Planas Vilá M, Cuerda Compés MC, Luengo Pérez LM. Nutrición artificial domiciliaria. En: Gil Hernández A, director. Tratado de nutrición, tomo IV; Nutrición Clínica. $2^{a}$ ed. Madrid: Médica Panamericana; 2010. p. 201-23.

2. Ministerio de Sanidad y Consumo. Guía de nutrición enteral domiciliaria en el Sistema Nacional de Salud. Madrid: Ministerio de Sanidad y Consumo, 2008.

3. Pedrón-Giner C, Puiggrós C, Calañas A, Cuerda C, García-Luna PP, Irles JA, et al; Grupo NADYA-SENPE. Registro del grupo NADYA-SENPE de Nutrición Enteral Domiciliaria en España en el año 2008. Nutr Hosp. 2010;25(5):725-9. DOI: 10.3305/nh.2012.27.1.5584; PMID: 21336427

4. Wanden-Berghe C, Puiggrós C, Calañas A, Cuerda C, García-Luna PP, Rabassa-Soler A, et al; Grupo NADYA-SENPE. Registro español de nutrición enteral domiciliaria del año 2009; Grupo NADYA-SENPE. Nutr Hosp. 2010; 25(6):959-63. DOI: 10.3305/nh.2015.31.6.8983; PMID: 21519767

5. Cuerda C, Parón L, Planas M, Gómez C, Moreno JM; Grupo NADYA-SENPE. Presentación del nuevo registro español de pacientes con nutrición artificial domiciliaria. Nutr Hosp. 2007;22(4):491-5. PMID: 17650891

6. Wanden-Bergue C, Sanz-Valero J, Culebras J; Red Mel-CYTED. Información en Nutrición Domiciliaria: la importancia de los registros. Nutr Hosp. 2008;23(3):220-5. PMID: 18560698

7. Wanden-Bergue C, Pérez de la Cruz A, Lobo G, Calleja A, Gómez C, Zugasti A, et al; Grupo NADYA-SENPE. El registro de Nutrición Artificial Domiciliaria y Ambulatoria de la Sociedad Española de Nutrición Parenteral y Enteral; análisis DAFO. Nutr Hosp. 2012;27(4):1357-60. DOI: 10.3305/nh.2012.27.4.5912; PMID: 23165586

8. Luengo Pérez LM, Chicharro ML, Cuerda C, García Luna PP, Rabassa Soler A, Romero A, et al; Grupo NADYA-SENPE. Registro de Nutrición Enteral Domiciliaria en España en el año 2007. Nutr Hosp. 2009;24(6):655-60. DOI:10.3305/nh.2009.24.6.4548; PMID: 20049367

9. Frías L, Puiggrós C, Calañas A, Cuerda C, García-Luna PP, Camarero E, et al; Grupo NADYASENPE. Nutrición enteral domiciliaria en España: registro NADYA del año 2010. Nutr Hosp. 2012;27(1):266-9. DOI: 10.1590/S0212-16112012000100035; PMID: 22566332

10. Villar Taibo R, Martínez Olmos MA, Rodríguez Iglesias MJ, Fernández Rodríguez E, Prieto Tenreiro A. Home artificial nutrition in a sanitary area of Galicia (Spain): descriptive study and proposals for the future. Nutr Hosp. 2008;23(5):433-8. PMID: 19160893

11. Leyes P, Forga MT, Montserrat C, Coronas R. Nutrición enteral domiciliaria. Casuística del Hospital Clínico de Barcelona. Nutr Hosp. 2001;16(5):152-6. PMID: 11702417

12. Vidal Casariego A, Ballesteros MD, Calleja Fernandez A, Brea Laranjo C, Fariza Vicente E, Cano Rodríguez I. Evolución de la nutrición enteral domiciliaria en el Área de Salud de León. Nutr Hosp. 2009;24(Supl 1):51.

13. De Luis DA, Izaola O, Cuellas LA, Terroba MC, Cabezas G, De la Fuente B. Experience over 12 years with home enteral nutrition in a healthcare área of Spain. J Hum Nutr Diet. 2013;26(1):3944. DOI: 10.1111/jhn.12081; PMID: 23651049

14. Pérez Méndez LF, García-Mayor RV; Grupo de Trabajo de la Sociedad Gallega de Nutrición y Dietética. Situación actual de la nutrición enteral domiciliaria en Galicia. Estudio multicéntrico. Nutr Hosp. 2001;16(6):257-61. PMID: 11840589 
15. Chicharro L, Planas M, Pérez-Portabella C, Vélez C, San José A. El soporte nutricional en el ámbito de la hospitalización a domicilio. Nutr Hosp. 2009;24(1):93-6. PMID: 19266120

16. Castaño Escudero A, Pérez Gabarda ME. Evolución de la nutrición enteral domiciliaria en Madrid 2002-2007. Nutr Hosp. 2009;24(2):218-25. PMID: 19593495

17. Castelló-Botía I, Wanden-Berghe C, Sanz-Valero J. Artificial Nutritional Support Registries: systematic review. Nutr Hosp. 2009;24(6):711-6. DOI:10.3305/nh.2009.24.6.4556; PMID: 20049375

18. Moreno Villares JM. La práctica de la nutrición artificial domiciliaria en Europa. Nutr Hosp. 2004;19(2):59-67. PMID: 15049406

19. Santarpia L, Pagano MC, Pasanisi F, Contaldo F. Home artificial nutrition: An update seven years after de regional regulation. Clin Nutr. 2014;33(5):872-8. DOI: 10.1016/j.clnu.2013.10.005; PMID: 24182767

20. Pironi L, Candusso M, Biondo A, Bosco A, Castaldi P, Contaldo F, et al; Italian Society for Parenteral and Enteral Nutrition Executive Committee. Prevalence of home artificial in Italy in 2005 a survey by the Italian Society for Parenteral and Enteral Nutrition (SINPE). Clin Nutr. 2007;26(1):123-32. DOI: 10.1016/j.clnu.2006.07.004; PMID: 16938366

21. Smith T, Micklewright A, Hirst A, Stratton R, Baxter J, Zeraschi S (editors). Annual BANS Report, 2011: Artificial Nutrition Support in the UK 2000-2010 [monograph on the Internet]. Redditch, Worcs: The British Association for Parenteral and Enteral Nutrition; 2011 [cited 24 nov 2016]. Available from: https://goo.gl/24jpWl

22. Lescut D, Dauchet L, Leroy M, Danel N, Alix E, Bertin E, et al. Incidence et prevalence de la nutrition entérale à domicile en France. Nutr Clin Met. 2013;27(4):171-7. DOI: 10.1016/j.nupar.2013.09.010

23. Matía P, Cuerda C, Burgos R, Álvarez J, Luengo LM, Calleja A, et al. Nutrición Enteral Domiciliaria. Registro Nacional 2012. Nutr Hosp 2013;28(Supl.3):53-4. DOI: 10.3305/nh.2013.28. sup3.6641 